Submitted to: Nucl. Instr. Meth. A, Proc. SRI91 C.onf. Synchr. Rad. (Baton Rouge, 1991)

\title{
$X$-ray intensity interferometer for undulator radiation
}

E. GLUSKIN, I. MCNULTY, and P.J. VICCARO

Advanced Photon Source

Argonne National Laboratory, Argonne, IL 60439, USA

The submitted manuscript has been authored
by a contractor of the U.S. Goremment
under contract No. W.31-109ENG-38.
Accordingly, the U.S. Government retains a
nonexclusive, loyalty-free license to publish
or repoduce the published form of this
contribution, or allowiv others to do so, for
U.S. Government purposes.

M. R. HOWELLS ${ }^{\dagger}$

Advanced Light Source

Lawrence Berkeley Laboratory, Berkeley, CA 94720, USA
ANL/CP- -76025

DE92 015184

\begin{abstract}
Intensity interferometry is well established with visible light but has never been demonstrated with $x$-radiation. We propose to measure the transverse coherence of an x-ray beam, for the first time, using the method of Hanbury Brown and Twiss. The $x$-ray interferometer consists of an array of slits, a grazing incidence reflective beamsplitter, a pair of fast multichannel plate detectors and a broadband, low-noise correlator circuit. The NSLS $X_{1}$ or $X 13$ soft $x$-ray undulator will supply the partially coherent $x$-rays. We are developing this technique to characterize the coherence properties of $x$-ray beams from high brilliance insertion devices at third-generation synchrotron light facilities such as the Advanced Photon Source and the Advanced Light Source.
\end{abstract}

\section{Introduction}

Hanbury Brown and Twiss (HBT) intensity interferometry was first applied optically to measurement of the angular sizes of stars [1]. It is potentially suited to characterization of the coherence properties of $x$-ray beams prodsiced by high brilliance undulators [2-4]. The vertical phase space of undulator emission approaches the diffraction limit at moderi low-emittance storage rings, therefore, determination of the source extent and shape is of considerable interest for both $x$-ray and electron beam diagnostics, and for coherencedependent applications such as $x$-ray microfocusing [5] and holography [6].

Turrently at the European Synchrotron Radiation Facility, BP220, 38043 Grenoble, France.

DASTHIBUTION GI: TIHS DOCUMENT IS UNLIMITED 
HBT interferometry is particularly promising for $x$-ray source characterization because it is capable of diffraction-limited resolution. Moreover, intensity interferometry is much more tolerant of optical path-length mismatches and of optics figure and surface finish imperfections than amplitude interferometry.

An intensity interferometer determines the coherence between two transversely separated points in a photon beam by cross-correlating the intensity fluctuations detected at the two points. A spectrally brilliant source is necessary because the degree of correlation depends on the square of the number of quanta per unit bandwidth. The NSLS soft $x$-ray undulator (SXU) beamlines, $X 1$ and $X 13$, are the most brilliant continuous soft $x$-ray sources now operating $[7,8]$, thus thev are uniquely suited to a demonstration of this technique in the $x$-ray region.

We plan to measure the transverse (spatial) coherence of the $X 1$ or $X 13$ undulator beam in the vertical plane using an intensity interferometer designed for the soft $x$-ray region. Based on the substantial spectral brilliance of the devices $\left(6-18 \times 10^{16}\right.$ photons $/ \mathrm{mm}^{2} / \mathrm{mrad}^{2} / \mathrm{s} / 0.1 \%$ BW at a wavelength of $\left.3.5 \mathrm{~nm}\right)$, we calculate that a coherence measurement is feasible on time scales of several minutes using an interferometer with a time resolution of a few nanoseconds. In this paper we discuss our experimental approach, pertinent source characteristics for the X1 SXU and several third-generation undulators, and the anticipated performance of the interferometer at the $X 1 A$ beamline.

\section{Experiment}

Our approach is similar to the analog experiments performed by Hanbury Brown and Twiss witl. laboratory visible light sources [10]. The photon counting method is not practical in the $x$-ray region with presently available sources and monochromators. In this HBT experiment, two analog (current-mode) $x$-ray detectors with slit shaped apertures are deployed in a munochromatized undulator beam (see fig. 1). The detector signals feed a correlator circuit in which the time-varying components are amplified then delivered to a linear multiplier and low-pass filter that take their time-averaged product. The correlator output is nonzero when the detector current fluctuations are sufficiently correlated. The fluctuations in the incident beam occur naturally on the scale of its coherence time, $t_{c}=\lambda^{2} / c \Delta \lambda$. Since electionically achievable bandwidths are much smaller than $1 / t_{c}$ for usual $x$-ray monochromators, 
obtaining a statistically significant correlation requires averaging for many coherence time intervals. The temporal resolution of the detectors, amplifiers, and multiplier should, therefore, be as short as possible to minimize the measurement time.

The sizes of the slits define the interferometer acceptance, and their spacing determines the relative phase difference introduced between the Fourier components of the waves incident on the detectors. The correlation measured for a particular slit separation is proportional to the modulus of $\mu_{12}$, the complex degree of coherence of the beam. The beam's coherence width is mapped by recording the integrated $\mathrm{cc}$ relatc: output for several slit spacings ranging from zero separation, where the correlation is greatest, to the point where the measurable correlation becomes lost in the background. A plot of the correlation as a function of slit separation gives $\left|\mu_{12}\right|$ over the beam dimensions, from which the source size can be found according to the Van Cittert-Zeinike theorem [9]. Provided the source distribution is symmetrical, its shape is determined by taking the Fourier transform of $\left|\mu_{12}\right|$.

\section{Source properties}

Table 1 gives the source dimensions for the $\mathrm{X} 1 \mathrm{SXU}$. We will restrict our attention to the vertical plane, because very little of the flux is coherent in the horizontal plane (the eleciron beam dominates the horizontal phase space of the photon beam) and the $\mathrm{X} 1 \mathrm{~A}$ beamline spherical grating monochromator disperses horizontally. The vertical coherence width at the experiment is defined $23 \mathrm{~m}$ away by the source, while the monochromator exit slit defines the horizontal coherence region approximately $3 \mathrm{~m}$ away. The interferometer will therefore be oriented such that it is insensitive to horizontal intensity fluctuations. The $X 1$ and $X 13$ SXU beams are spatially stabilized in two dimensions by beam position monitor feedback control systems [8].

From table 1, it is clear that a significant fraction of the flux in the SXU beam is coherent along the vertical axis. On the other hand, the SXU beam is sufficiently naturally collimated vertically that its coherence angle is comparable to the beam divergence making intensity correlations difficult to measure near the beam's vertical angular extremities. Hence, it may be necessary to narrow the coherence angle by increasing the horizontal to vertical coupling of the stored electron beam, thereby widening its vertical extent. 
The signal-to-noise ratio applicable to intensity interferometry depends critically on the source degeneracy parameter $\delta$, the number of photons emitted per phase space volume per coherence interval $[11,12]$. For optical lasers $\delta$ is huge. By contrast, it is usually less than unity even for the most brilliant $x$-ray sources. The source degeneracy parameter can be calculated for undulators via

$$
\delta=B \frac{\lambda^{3}}{4 c}
$$

where $\lambda$ is the wavelength, $C$ is the velocity of light, and the spectral brilliance $B$ is defined per unit fractional bandwidth (note $B$ is commonly quoted per $0.1 \%$ BW). Eq. 1 characterizes the source irrespective of the interferometer acceptance. But, the strongly differing vertical and horizontal coherence properties of undulator beams suggest an alternative treatment. Assuming the interferometer accepts the entire horizontal extent as well as the vertically coherent portion of the beam, the effective vertical degeneracy parameter

$$
\begin{aligned}
& \delta_{y}=B \frac{\lambda^{2}}{2 c} \Sigma_{x} \Sigma_{x^{\prime}} \\
& =1.67 \times 10^{-18} \quad B\left[\mathrm{~s} \cdot \mathrm{mm}^{2} \cdot \mathrm{mrad}^{2} .0 .1 \% B W\right]^{-1} \\
& \times \lambda^{2}[\mathrm{~nm}] \Sigma_{x}[\mathrm{~mm}] \Sigma_{x^{\prime}}[\mathrm{mrad}]
\end{aligned}
$$

is the relevant figure of merit, where $\Sigma_{x}$ and $\Sigma_{x^{\prime}}$ are the horizontal source size and divergence. By contrast to the cubic dependence of $\delta$ on $\lambda, \delta_{y} \propto \lambda^{2}$. Corisequently, the effective vertical degeneracies of hard $x$-ray relative to soft $x$ ray undulators vary much less than their respective source degeneracy parameters, which may span five or more orders of magnitude. Table 2 compares $\delta_{y}$ for the $X 1$ SXU with several high brilliance $x$-ray sources under construction. 


\section{The interferometer}

The interferometer must be compact to accomodate the small SXU beam footprint, a few square $\mathrm{mm}$, at the beamline end-station. Our instrument selects two rays from the incident $x$-ray beam with an array of tiny slits, then splits them with a slender prism-shaped grazing incidence mirror (illustrated schematically in fig. 2). Due to the phase insensitivity of the HBT technique, it is not necessary for the mirror to possess highly flat and smooth surfaces. After the two beams have diverged sufficiently they can be sensed by detectors of ordinary size.

The slit array contains several pairs of narrow apertures, 20-50 $\mu \mathrm{m}$ high by 1 $\mathrm{mm}$ across, with spacings ranging from $50 \mu \mathrm{m}$ to $2 \mathrm{~mm}$. These slit dimensions enable sampling of the beam's vertical coherence width $(1.3 \mathrm{~mm})$ with enough spatial resolution to map its extent, yet accept the full horizontal breadth of the beam. Each slit pair is selected by translating the array on a linear manipulator. The slit array can be produced by laser-drilling a thin metal substrate, which should be at least $10 \mu \mathrm{m}$ thick to isolate the two beams effectively. The straightness and parallelism of the slits need only be accurate to a few mrad.

The detectors are low-impedance, high-current microchannel plates (MCPs) chosen for short time resolution and wide dynamic range. The time response of the MCPs is minimized by using low resistance gold anodes, and their soft $x$-ray quantum efficiency is enhanced by coating the photocathodes with a thin layer of Csl or Cul. The interferometer is operated in an ultrahigh vacuum environment to protect the mirror, photocathodes and MCPs from contamination and to minimize $x$-ray absorption in the beam path.

The interferometer circuitry will be optimized for low noise and high speed; a time resolution of $3 \mathrm{~ns}$ is a reasonable objective using commercial linear electronics. In order for the experiment to succeed the correlator should have extremely little noise and be minimally susceptible to drift. A configuration designed to accomplish this using synchronous (i.e. lock-in) detection is diagrammed in fig. 3. Standard NIM modules perform the duties of the major functional blocks. An RF double-balanced mixer serves as the multiplier. This circuit essentially emulates that built by HBT [10]. However, in addition to a magnitude greater bandwidth, the hardware we have selected has lower noise, better stability, and is more easily reconfigurable.

The signal-to-noise ratio can be further optimized by taking advantage of the strict time structure of the source. Undulator radiation arrives in pulses reflecting the bunched nature of the electron beam. By ignoring random coincidences that 
occur between bunches it is possible to reduce the noise background significantly. This could be accomplished by gating the interferometer detectors and phase-locking the correlator to the bunch arrival time, which is dictated by the storage ring RF cavity frequency.

\section{Anticipated performance}

Based on the $x$-ray flux measured at the $X 1 A$ beamline [8] we can predict the mean detector photocurrent. At $150 \mathrm{~mA}$ current in the storage ring, the SXU typically produces a spectral flux of $2 \times 10^{11}$ photons $/ \mathrm{s} / 0.1 \% \mathrm{BW}$ at $\lambda=3.5 \mathrm{~nm}$, or about $2 \times 10^{-3}$ photons $/ \mathrm{s} / \mathrm{Hz}$. A pair of $20 \mu \mathrm{m} \times 1 \mathrm{~mm}$ interferometer slits accepts $6 \times 10^{-7} \mathrm{~mm}^{2} \cdot \mathrm{mrad}^{2}$ and samples approximately $3 \%$ of the vertical coherence width at the end of the beamline. The slits admit a flux of $4 \times 10^{10}$ photons/s accounting for the geometrical factors, a beamline efficiency of $6 \%$, and a $1 \%$ spectral bandpass provided by the $\mathrm{X} 1 \mathrm{~A}$ spherical grating monochromator. Estimating that a detective quantum efficiency (DQE) of $10 \%$ is achievable at this wavelength for the mirror plus MCPs, we find the primary photoemission current in each MCP is $2 \times 10^{9}$ photoelectrons/s. This is comparable to the photocurrents obtained by HBT [17] using photomultipliers and visible light. A interesting feature of HBT interferometry is that the intrinsic signal-to-noise ratio $\mathrm{S} / \mathrm{N}$ is independent of the optical bandwidth. Thus, the beam monochromaticity can be adjusted to match the total flux incident on the interferometer to the available detector dynamic range without unduly sacrificing the incident spectral flux.

The measurement time needed to achieve a desired $\mathrm{S} / \mathrm{N}$ is given by

$$
T=\frac{(S / N)^{2}}{D Q E} \frac{\tau_{0}}{\left(\varepsilon \eta \delta_{y}\right)^{2}\left|\mu_{12}\right|^{4}}
$$

where $\tau_{0}$ is the interferometer time resolution, $\varepsilon$ is the accepted fraction of the vertical coherence width, $\eta$ is the beamline efficiency, and the intensities in both interferon eter arms are taken to be equal. We wish to measure values of $\left|\mu_{12}\right|$ as small as 0.2 with a mean signal that is at least three times the rms noise ( $\mathrm{S} / \mathrm{N}=3$ ). Using $\varepsilon=3 \%, \eta=6 \%, \tau_{0}=3 \mathrm{~ns}$, and $\delta_{y}=0.2$, we obtain $\mathrm{T} \sim 1300 \mathrm{~s}$ by

eq. 3. The coherence measurement requires dramatically less time (or yields better $S / N$ ) when the slits are located adjacent to one another, since $\left|\mu_{12}\right| \rightarrow 1$. 


\section{Conclusion}

Currently, we are assembling the components for the HBT interferometer and will begin testing the completed device in early 1992. Successful extension of this technique to the $x$-ray region will significantly augment our ability to characterize the coherence properties of high brilliance undulator beams. This diagnostic tool is potentially valuable for $x$-ray and electron beam characterization at third-generation synchrotron radiation facilities such as the Advanced Photon Source and the Advanced Light Source.

\section{Acknowledgements}

This work supported by the U.S. Department of Energy, Office of Basic Energy Sciences, Division of Material Sciences, under contract number W-31109-ENG-38 (E.G., I.M. and P.J.V.), and contract number DE-AC03-76SF00098 (M.R.H.).

\section{DISCLAIMER}

This report was prepared as an account of work sponsored by an agency of the United States Government. Neither the United States Government nor any agency thereof, nor any of their employees, makes any warranty, express or implied, or assumes any legal liability or responsibility for the accuracy, completeness, or usefulness of any information, apparatus, product, or process disclosed, or represents that its use would not infringe privately owned rights. Reference herein to any specific commercial product, process, or service by trade name, trademark, manufacturer, or otherwise does not necessarily constitute or imply its endorsement, recommendation, or favoring by the United States Government or any agency thereof. The views and opinions of authors expressed herein do not necessarily state or reflect those of the United States Government or any agency thereof. 
[1] R. Hanbury Brown and R.Q. Twiss, "A Test of a New Type of Stellar Interierometer on Sirius", Nature 178, 1046 (1956)

[2] E.V. Shuryak, "Two-photon correlations in synchrotron radiation as a method of studying the beam", Sov. Phys. JETP 40, 30 (1975)

[3] M.R. Howells, "The X-Ray Hanbury-Brown and Twiss Intensity Interferometer: a New Physics Experiment and A Diagnostic for Both XRay and Electron Beams at Light Sources",

ALS Technical Report, LSBL 27 (2 February 1989)

[4] E. Gluskin, "Intensity Interferometry and its Application to Beam Diagnostics", Proc. Particle Accel. Conf. (San Francisco, 1991) in press

[5] W.-B. Yun, P.J. Viccaro, J. Chrzas, and B. Lai, "Coherent hard x-ray focusing optics and applications", Proc. 4th Intl. Conf. on Synchr. Rad. Instr. (Chester, July 1991)

[6] I. McNulty, J. Kirz, C. Jacobsen, M.R. Howells and E. Anderson, "First Results with A Fourier Transform Holographic Microscope", A. Michette, G. Morrison and C.Buckley,eds., X-Ray Microscopy III (Springer-Verlag, Berlin, 1991) in press

[7] H. Rarback, C. Jacobsen, J. Kirz, and I. McNulty, "The Performance of the NSLS Mini-Undulator", Nucl. Instr. Meth. A266, 96 (1988)

[8] H. Rarback, et al., "Coherent Radiation for X-Ray Imaging -- The Soft XRay Undulator and the X1A Beamline at the NSLS", J. X-Ray Sci. Tech. 2, 274 (1990) 
[9] M. Born and E. Wolf, Principles of Optics, (Pergamon, Oxford, 1980) pp. $508-513$

[10] R. Hanbury Brown and R.Q. Twiss, "Interferometry of the intensity fluctuations in Light II. An experimental test of the theory for partially coherent light", Proc. Royal Soc. A243, 291 (1958)

[11] L. Mandel, "Fluctuations of Light Beams", E. Wolf, ed., Progress in Optics V (North-Holland, Amsterdam, 1963) pp. 181-248

[12] J.W. Goodman, Statistical Optics (Wiley, New York, 1985) pp. $481-490,501-510$

[13] Elettra Conceptual Design Report (Sincrotrone Trieste, Trieste, 1989) p. IV-11

[14] An ALS Handbook (Advanced Light Source, Berkeley, 1989) p. 40

[15] Foundation Phase Report (European Synchrotron Radiation Facility, Grenoble, 1987) p. CIV-305

[16] G.K. Shenoy, P.J. Viccaro, and D.M. Mills, Characteristics of the 7-GeV Advanced Photon Source: A Guide for Users (Argonne National Laboratory, Argonne, 1988) p. 24

[17] R. Hanbury Brown and R.Q. Twiss, "The Question of Correlation between Photons in Coherent Light Rays", Nature 178, 1447 (1956) 
Tables

\begin{tabular}{|lc|rc|}
\hline Horizontal size & $\Sigma_{x}$ & 390 & $\mu \mathrm{m}$ \\
Vertical size & $\Sigma_{y}$ & 20 & $\mu \mathrm{m}$ \\
Horizontal divergence & $\Sigma_{x^{\prime}}$ & 250 & $\mu \mathrm{rad}$ \\
Vertical divergence & $\Sigma_{y^{\prime}}$ & 70 & $\mu \mathrm{rad}$ \\
Horizontal coherence angle & $\Omega_{x}$ & 3 & $\mu \mathrm{rad}$ \\
Vertical coherence angle & $\Omega_{y}$ & 56 & $\mu \mathrm{rad}$ \\
\hline
\end{tabular}

Table 1. Source properties of the NSLS $X 1$ soft $x$-ray undulator for $\lambda=3.5 \mathrm{~nm}$. The rms electron beam source sizes and divergences are summed in quadrature with the intrinsic (diffiaction-limited) source sizes and divergences to obtain the values shown. In one dimension, pairs of points in the beam for which $\left|\mu_{12}\right|>0.84$ lie within an angle $\Omega<\lambda / \pi \Sigma$, where $\Sigma$ is the rms source size.

\begin{tabular}{|c|c|c|c|}
\hline Source & Brilliance & $\lambda(\mathrm{nm})$ & $\delta_{y}$ \\
\hline NSLS SXU & $2 \times 10^{17}$ & $2-5$ & $0.13 \cdot 0.8$ \\
\hline ELETTRA $U_{2}$ & $3 \times 10^{18}$ & $2-5$ & $0.19-1.2$ \\
\hline ALS U3.9, U5.0, U8.0 & $3 \times 10^{18}$ & $1-10$ & $0.08-8.0$ \\
\hline ESRF U 5,5, U3.5 & $2 \times 10^{18}$ & $0.1-1$ & $0.0003-0.013$ \\
\hline APS U-A, U-C & $2 \times 10^{18}$ & $0.1-1$ & $0.0003-0.03$ \\
\hline
\end{tabular}

Table 2. Estimated effective vertical degeneracy parameters for high brilliance undulator sources at various synchrotron radiation facilities $[8,13-16]$. Source brilliance is given for the machine design current in units of photons $/ \mathrm{s} / \mathrm{mm}^{2} / \mathrm{mrad}^{2} / 0.1 \%$ BW. 
Figure Captions

Fig. 1. Optical geometry for measuring the spatial coherence of an undulator beam by HBT intensity interferometry. The monochromator is presumed to be a standard undulator beamline component. The slits and detectors are aligned to the monochromatized beam; the correlator electronics are located nearby.

Fig. 2. Lateral view of the intensity interferometer (schematic, not to scale). The partially coherent $x$-ray beam enters the slits at top. X-rays passing through the slits are deflected at grazing incidence by a wedge-shaped mirror and detected by MCPs situated to each side of the optical axis. The MCP output signals are collected by coaxial $50 \Omega$ anodes, preamplified, then transmitted by shielded cable to the correlator.

Fig. 3. Block diagram of the HBT correlator. The first two mixers (at left) chop the detector output signals with square-wave envelopes supplied by local oscillators at frequencies $f_{1} \neq f_{2}$. The fast mixer (center) performs the correlation operation and the linear gates (at right) demodulate the correlator output at the two chopping frequencies. The degree of correlation is recorder' by an integrating latch and counter following the low-pass filters at the end of the signal chain. 
Fig. 1
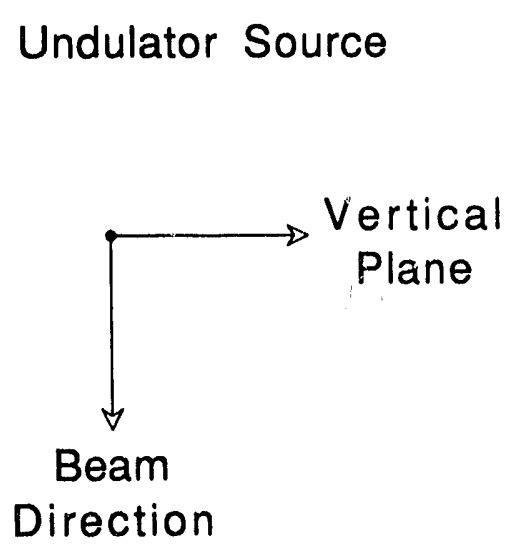

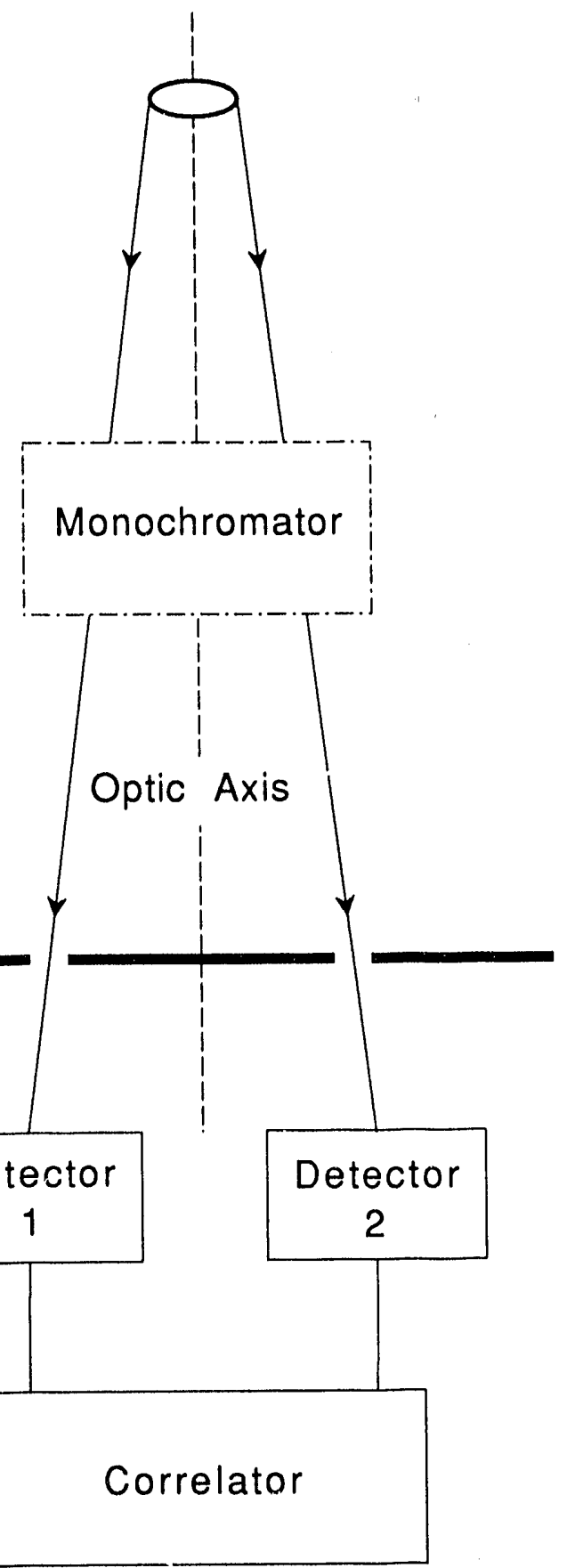




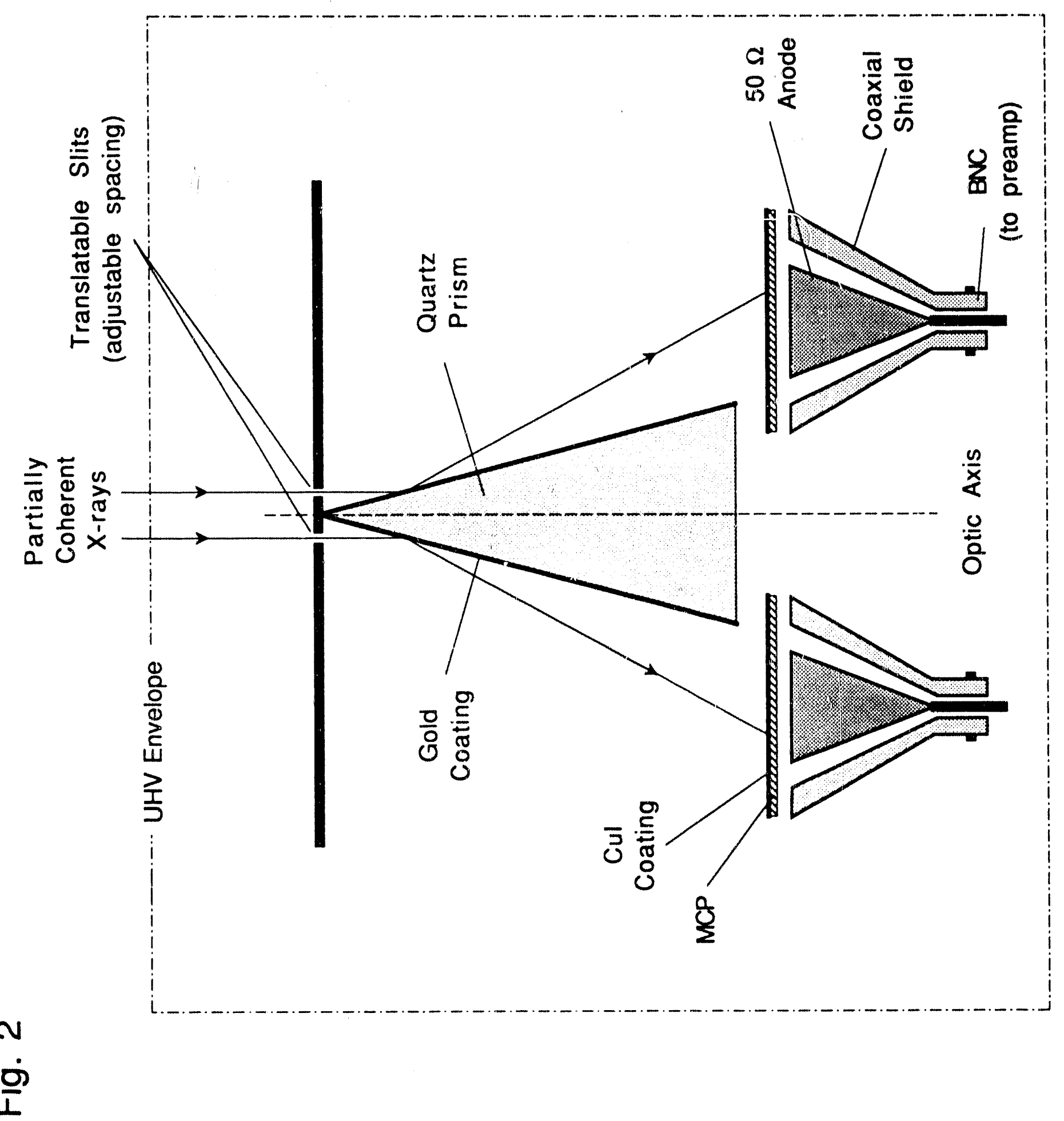




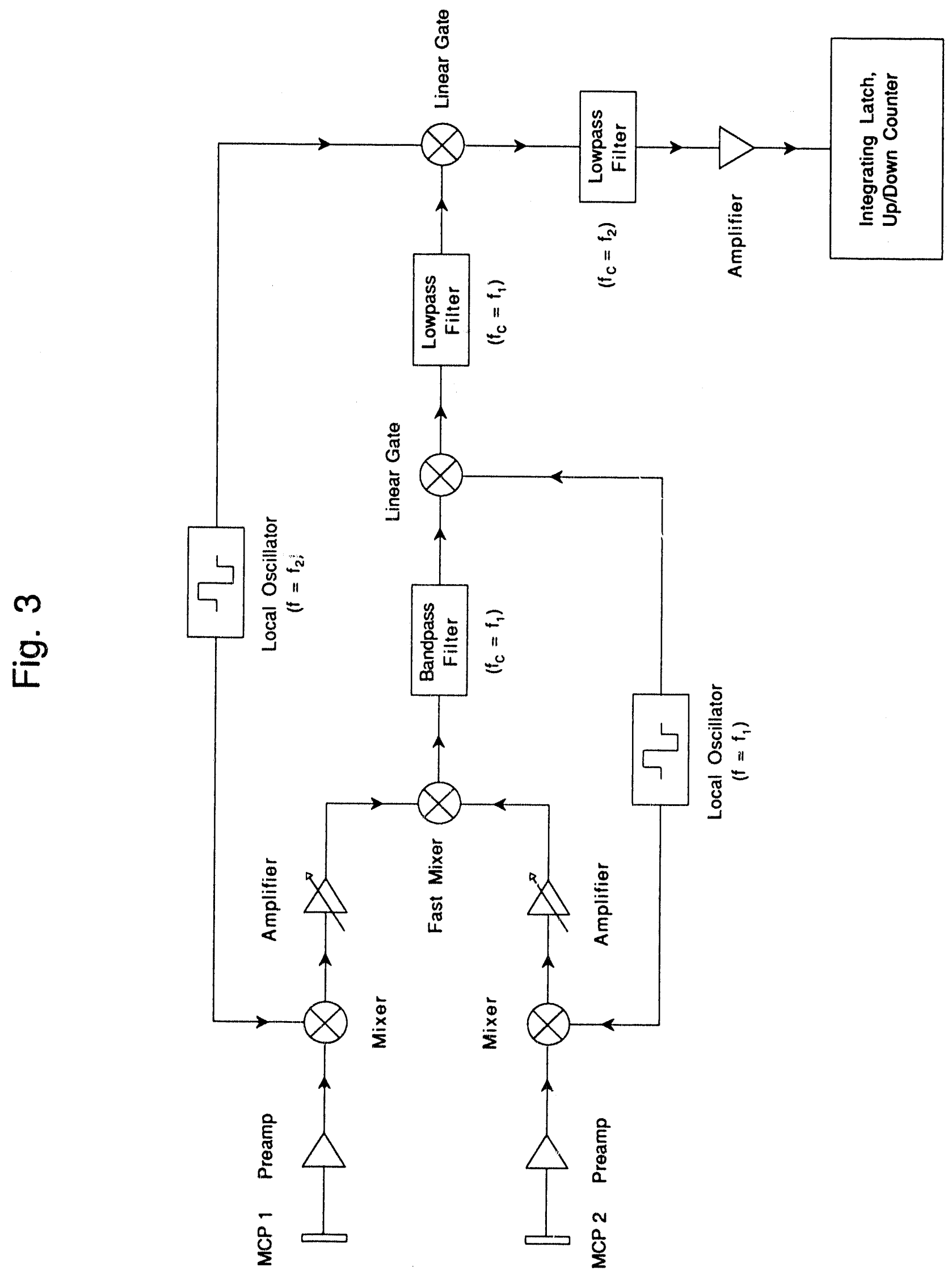



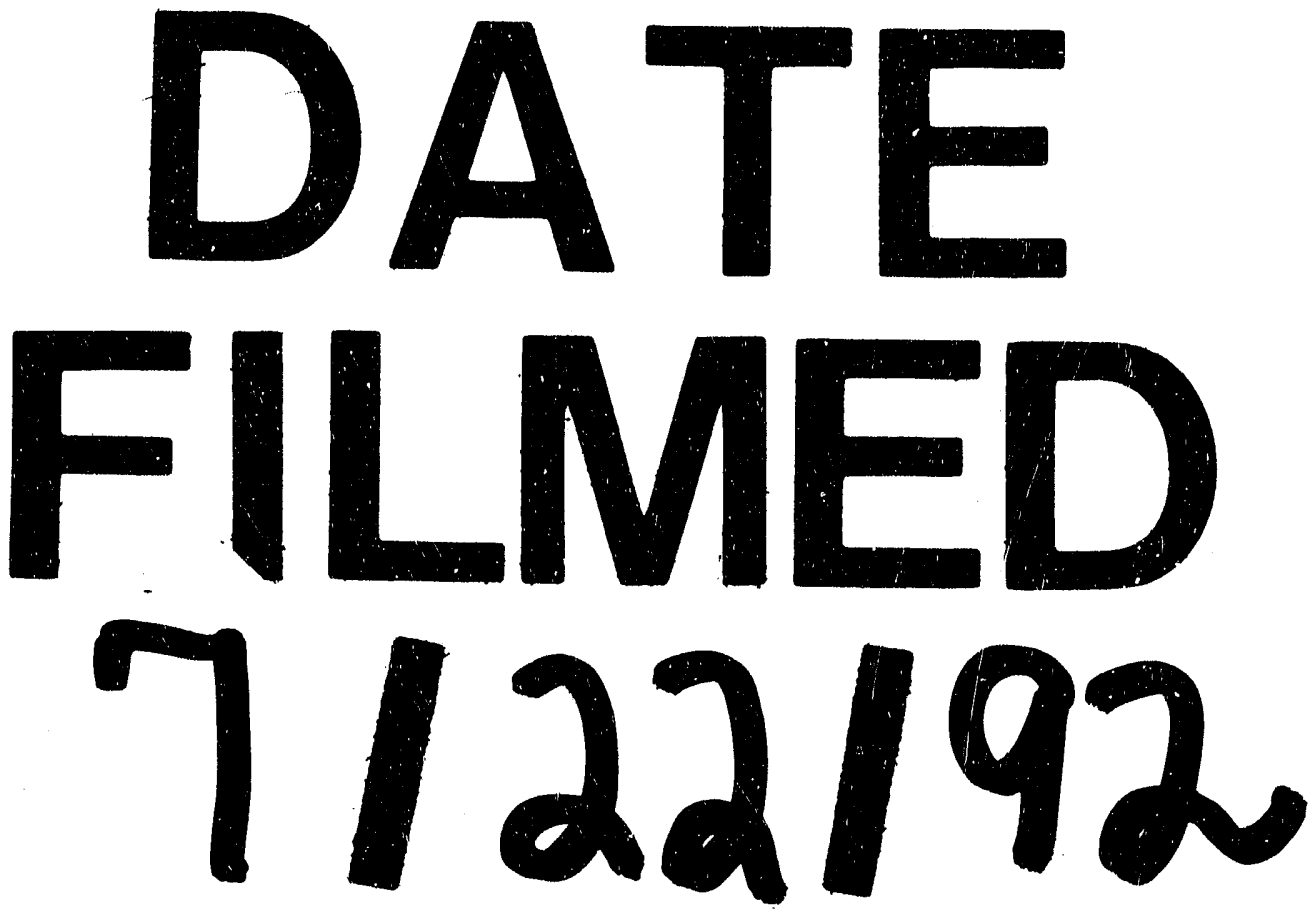
